\section{Poison pen letters}

\section{By Lev Osherovich, Senior Writer}

Researchers at The University of Queensland were using RNAi to knock down tumor-specific proteins when they encountered an unexpected finding: the RNAi itself stimulated immunity against tumor antigens. The work suggests RNAi could be used to flag cancer cells for detection by the immune system, potentially creating a new strategy for therapeutic cancer vaccination. ${ }^{1}$

Murine tumor cells that had been transformed with human papillomavirus (HPV), which causes cervical cancer, were treated with small hairpin RNAs designed to inactivate viral transcripts. The researchers found that the viral transcripts targeted by shRNAs were not totally destroyed, as would be expected based on previous shRNA studies. Instead, small fragments of these transcripts underwent translation, producing tiny fragments of HPV proteins. These fragments, in turn, were presented as surface antigens by the tumor cells.

"There's a hidden mechanism by which shRNA leaves messages cleaved but not destroyed," said Nigel McMillan, associate professor of cancer, immunology and metabolic medicine at the University of Queensland. "We've found that some of these messages turn out to be translated, generating epitopes that are expressed in cancer cells only."

McMillan's team went on to show that tumor cells bearing these surface epitopes elicited an immune response that protected mice. Mice injected with tumor cells treated ex vivo with anti-HPV shRNAs had smaller tumors than mice given untreated control tumor cells. Untreated tumor cells that were subsequently injected into the mice failed to take hold, presumably due to the animals' acquired immune response against tumor antigens.

Results were published in Proceedings of the National Academy of Sciences.

Altogether, the findings suggest that shRNA leads to the production of immunogenic protein fragments that alert the immune system to the presence of tumor cells.

McMillan believes that the adaptive immune system is critical for the process. "When we repeated these experiments with immunocompromised mice, it didn't work," he told SciBX.

The study presents a new use of RNAi to elicit an indirect protective effect rather than to simply kill tumor cells, said Ian MacLachlan, CSO of RNAi delivery company Tekmira Pharmaceuticals Corp.

"Typically when one uses an RNAi-mediated means of affecting tumor growth or progression, you're knocking down a tumor-related target to slow growth," said MacLachlan. "Here, they're trying to elicit an immune response against the tumor."

\section{Raise the alarm}

The RNAi approach for priming an immune response to tumors may have one major advantage over protein-based cancer vaccines: the ability to target a wide range of cancer antigens in a single formulation. The breadth of coverage is important for overcoming antigenic escape by rapidly mutating tumors.

"Cancers will mutate away target sites as fast as they can, so we should have three or four targets," said McMillan.

Although cancer vaccines with multiple proteins have been attempted, selecting and formulating the right tumor antigens is technically challenging and varies between indications. In contrast, the specific targets of an RNAi vaccine could be customized for each patient based on the tumor's gene expression profile.

An RNAi vaccine could also be more useful for personalized tumor therapy than protein vaccines because making customized RNAi cocktails based on an individual patient's tumor protein expression patterns is, in principle, easier than formulating complex mixtures of recombinant proteins.

However, the RNAi approach faces many of the same hurdles as protein-based vaccines, such as how and when to deliver the vaccine and how to measure efficacy.

Two companies have recently reported promising results with their respective cancer vaccines. Dendreon Corp.'s Provenge sipuleucel-T met its survival endpoint in a Phase III prostate cancer trial. ${ }^{2}$ Last month, Antigenics Inc. reported data from an extension of a Phase III trial that showed increased survival in kidney cancer patients receiving the company's Oncophage vitespen.

Oncophage uses heat shock protein GP96 and associated peptides isolated from the patient's tumor. Provenge is composed of autologous dendritic cells loaded with a fusion protein of prostatic acid phosphatase and an immunostimulatory cytokine.

Andrew Parsa, associate professor of neurological surgery at the University of California, San Francisco, described the RNAi vaccination method as less direct than vaccinating with tumor-associated proteins such as GP96.

"This is the long road to doing what the heat shock protein vaccine does," said Parsa.

Parsa is primary investigator on a Phase II trial of Oncophage for primary glioblastoma, which the company said is enrolling patients. A Phase I trial of Oncophage in recurrent, high-grade glioma showed an increase in mean survival time, according to Antigenics.

Parsa noted that Oncophage is aided by the presence of specific receptors for tumor-derived GP96 on immune cells. In contrast, an RNAi-based approach still would require tumor-derived protein fragments to be taken up by professional antigen-presenting cells such as dendritic cells.

"Heat shock proteins are a natural part of our immune response. They have a lock and key mechanism for getting antigens to the presenting cells," said Parsa. "Almost every other approach requires there to be an almost random mechanism" for delivering the vaccine to the correct immune cells. 


\section{TARGETS \& MECHANISMS}

\section{Running interference}

McMillan now plans to optimize the RNAi formulation to make the vaccine suitable for direct intratumoral injection. His group is also working on a topical formulation of the HPV vaccine.

"Our next step is to do this with small interfering RNAs, which are a lot easier for safety and efficacy" than the shRNAs used in the study, he said.

The biggest test of the RNAi approach will be to see whether knocking down other tumor-linked genes besides HPV transcripts can cause a protective immune response. McMillan noted that targeting host-derived cancer proteins could potentially elicit an autoimmune reaction.

The discoveries are the subject of a pending patent, which is available for licensing from Uniquest Pty Ltd., the University of Queensland's commercial enterprise development company.
Osherovich, L. SciBX 2(23); doi:10.1038/scibx.2009.931

Published online June 11, 2009

\section{REFERENCES}

1. Gu, W. et al. Proc. Natl. Acad. Sci. USA; published online May 12, 2009; doi:10.1073/pnas.0812085106

Contact: Nigel A.J. McMillan, The University of Queensland, Brisbane, Queensland, Australia

e-mail: n.mcmillan@uq.edu.au

2. Flanagan, M. BioCentury 17(18), A1-A5; April 20, 2009

COMPANIES AND INSTITUTIONS MENTIONED

Antigenics Inc. (NASDAQ:AGEN), New York, N.Y. Dendreon Corp. (NASDAQ: DNDN), Seattle, Wash.

Tekmira Pharmaceuticals Corp. (TSX:TKM), Burnaby, British

Columbia, Canada

Uniquest Pty Ltd., Brisbane, Queensland, Australia

University of California, San Francisco, Calif.

The University of Queensland, Brisbane, Queensland, Australia 